\title{
Comparison of Accelerated Solvent Extraction (ASE) and Energized Dispersive Guided Extraction (EDGE) for the Analysis of Pesticides in Leaves
}

\author{
Ashlie D. Kinross, ${ }^{1}$ Kimberly J. Hageman, ${ }^{*}{ }^{*}$ William J. Doucette ${ }^{2}$, Alexandria L. Foster ${ }^{1}$ \\ ${ }^{1}$ Department of Chemistry and Biochemistry, Utah State University, USA \\ ${ }^{2}$ Department of Civil and Environmental Engineering, Utah State University, USA \\ Corresponding Author \\ *To whom correspondence should be addressed: \\ E-mail: kim.hageman@usu.edu; \\ Telephone: 1-435-797-0114
}

\begin{abstract}
:
Various techniques have been evaluated for the extraction and cleanup of pesticides from environmental samples. In this work, a Selective Pressurized Liquid Extraction (SPLE) method for pesticides was developed using a Thermo Scientific Accelerated Solvent Extractor (ASE). This instrument was compared to the newly introduced (2017) extraction instrument, the Energized Dispersive Guided Extraction (EDGE), which combines Pressurized Liquid Extraction (PLE) and dispersive Solid Phase Extraction (dSPE). We first optimized the SPLE method using the ASE instrument for pesticide extraction from alfalfa leaves using layers of Florisil and graphitized carbon black (GCB) downstream of the leaf homogenate in the extraction cell (Layered ASE method). We then compared results obtained for alfalfa and citrus leaves with the Layered ASE method to those from a method in which the leaf homogenate and sorbents were mixed (Mixed ASE method) and to similar methods modified for use with EDGE (Layered EDGE and Mixed EDGE methods). The ASE and EDGE methods led to clear colorless extracts with low residual lipid weight. No significant differences in residual lipid masses were observed between the methods. The UV-Vis spectra showed that Florisil removed a significant quantity of the lightabsorbing chemicals but GCB was required to produce colorless extracts. Recoveries of spiked analytes into leaf homogenates were generally similar among methods, but in several cases, significantly higher recoveries were observed in ASE extracts. Nonetheless, no significant differences were observed among pesticide concentrations in field samples when calculated with the isotope dilution method in which labelled surrogates were added to samples before extraction. Extraction time using the ASE methods were $\sim 45$ minutes, which is $\sim 4.5$ times longer than the EDGE methods. The EDGE methods use $\sim 10 \mathrm{~mL}$ more solvent than the ASE methods. Based on these results, the EDGE is an acceptable extraction instrument and, for most compounds, the EDGE has a similar extraction efficiency to the ASE.
\end{abstract}




\section{Keywords:}

- Selective Pressurized Liquid Extraction

- Alfalfa Leaves

- Citrus Leaves

- Graphitized Carbon Black

- In-cell cleanup

- Semi-volatile Organic Compounds

\section{Introduction:}

Pesticides (including herbicides, insecticides, and fungicides) are commonly used in agricultural systems worldwide; however, concerns about their negative impacts on human health and the environment [1] require careful monitoring of their concentrations in plant tissues and other environmental matrices. The extraction step is a critical, but often challenging, step in the quantification of pesticides in plant materials. A number of approaches for extracting pesticides from plant materials have been used, including Pressurized Liquid Extraction (PLE) [2-8], Selective PLE (SPLE) [4,5], Quick, Easy, Cheap, Effective, Rugged, and Safe (QuEChERS) [3,79], Matrix Solid-Phase Dispersion (MSPD) [3,4,7,10], Soxhlet [2,3,6,8], and dispersive Solid Phase Extraction (dSPE) $[3,7,9,10]$.

The Energized Dispersive Guided Extraction $\left(\mathrm{EDGE}^{\circledR}\right)$ system was introduced by CEM Corporation in October 2017 and was developed to combine PLE with dSPE [11] and is claimed to be 'faster than Soxhlet, more automated than QuEChERS, and simpler than other solvent extraction systems' [12]. A brief description of the EDGE approach is included here; for further details, readers should consult the CEM website and EDGE manual. To perform an EDGE extraction, the sample and selected sorbents are added to an extraction cell that contains a filter at the bottom of the cell. The instrument transfers the cell into a sealed, sample chamber where specified volumes of solvents are added from the bottom to the gap between the cell and chamber walls ('Bottom Volume'). A smaller amount of solvent is also added to the cell from the top ('Top 
Volume'). The walls of the chamber are heated $\left(25-200{ }^{\circ} \mathrm{C}\right)$, causing the solvent to expand and disperse into the sample through the holes in the bottom of the cell. Because the system is sealed, temperatures above the solvent boiling point can be used and the volatilized solvent will not escape the cell (maximum pressure is $200 \mathrm{psi}$ ). Solvent is held in the cell for a selected amount of time, allowing analytes to partition into the solvent. The solvent and extracted analytes then drain through the filter into a collection vial. While a number of Application Notes for EDGE methods can be found on the CEM website [13], to the best of our knowledge, no peer-reviewed publications exist that evaluate EDGE or compare its performance to that of any of the well-known, trusted extraction approaches.

To evaluate the performance of the EDGE, we compared it to an SPLE method designed for use with a Thermo Scientific Dionex Accelerated Solvent Extractor (ASE). We used the ASE for this comparison because of similarities between EDGE and ASE instrumentation. SPLE has been described in detail in Subedi et al. [5]. In brief, SPLE is conducted by packing the sample and a layer of selected sorbents into a stainless steel extraction cell. The sorbents are included to retain compounds that interfere with instrument analysis of target compounds (i.e. in-cell cleanup). The extraction cells are transferred to an oven, solvent is added to the sealed cell, and the cell is heated $\left(40-200{ }^{\circ} \mathrm{C}\right)$. Since the system is closed, the solvent remains in the liquid phase while the pressure is maintained at a specified level during heating (typically to $1500 \mathrm{psi}$ ) using a pressure transducer and static valve. Elevated temperatures and pressures help to increase the efficiency of the extraction process [14]. After a selected hold time, the solvent and extracted analytes are transferred to a collection bottle. EDGE and ASE are similar in that they both use a robotic arm to move cells from a tray or rack of prepared cells, use elevated temperatures, and can perform incell cleanup by packing sorbents into the extraction cells. A critical difference is that the ASE 
instrument uses a much higher pressure in the cell. While the EDGE instrument does build up pressure (maximum $200 \mathrm{psi}$ ), the operating pressure is not set by the user. Instead, it is the result of the solvent expanding due to heat.

The first objective of this project was to optimize and validate an SPLE method using the ASE instrument for the extraction of a suite of 20 semi-volatile pesticides from citrus and alfalfa leaves. The second objective was to modify the optimized ASE method for use with the EDGE instrument and to compare the performance of the two instruments using various criteria. Because SPLE methods usually use layers of sorbent and sample in the extraction cell and because no investigations into the effects of layering versus mixing have been reported in the literature for either method, the third objective was to compare mixing and layering for each method. The four methods (Layered ASE, Mixed ASE, Layered EDGE, Mixed EDGE) were compared in terms of 1) extract color and extracted lipid mass, 2) lipid content analysis in extracts based on UV-Vis absorbance spectra, 3) recovery of spiked target pesticides, 4) pesticide concentrations in field samples, and 5) other extraction parameters.

\section{Materials and Methods:}

\subsection{Strategy Overview}

The first step was to optimize the in-cell cleanup of an ASE method for the extraction of pesticides in alfalfa leaves using a layered sorbent/leaf homogenate approach (the Layered ASE method). Optimization was achieved by determining the ideal masses of Florisil and graphitized carbon black (GCB) needed to obtain extracts that contained $<10 \mathrm{mg}$ of lipid and that were clear with minimal color. The $10 \mathrm{mg}$ lipid threshold was selected based on the recommendation in Lavin et al. [15]. Tests were then conducted to confirm that the Layered ASE method could also be used 
successfully with citrus leaves. Next, tests were conducted in which the sorbents and leaf homogenate were mixed, instead of layered, in the ASE extraction cell (Mixed ASE method). Finally, the ASE methods were modified for use with the EDGE (Layered EDGE and Mixed EDGE methods), using many of the same extraction parameters as in the ASE methods. UV-Vis absorbance spectra were obtained to determine how well selected sorbents removed colored matrix compounds. Spike and recovery experiments were conducted for a suite of 20 pesticides. Finally, pesticides were quantified in alfalfa and citrus field samples to compare concentrations measured using both methods.

\subsection{Materials, Chemicals, and Standards}

The following materials and chemicals were purchased from Thermo Fisher Scientific (Massachusetts, USA) and subsidiary companies: optima-grade ethyl acetate, $n$-hexane, Florisil (60-100 mesh size), sea sand, cellulose filter papers for the extraction cells, and diatomaceous earth (DE). GCB (ENVI-Carb Packing) was purchased from Sigma-Aldrich, Inc. (Missouri, USA). C4 Q-Disks were purchased from CEM Corporation (North Carolina, USA). Before use, Florisil, sand, and DE were baked at $565^{\circ} \mathrm{C}$ for 30 minutes to remove potential semi-volatile contaminants.

Twenty target pesticides were used in the spike and recovery experiments. Details about the pesticide classes investigated are provided in Table S1. Chlorpyrifos, cis-chlordane, dichlorodiphenyldichloroethane (o,p'-DDD and p,p'-DDD), dieldrin, endosulfan I, s-ethyl dipropylthiocarbamate (eptam), fenpropathrin, flupyradifurone, malathion, molinate, tolfenpyrad, triallate, and trifluralin were purchased from Sigma-Aldrich, Inc. (Missouri, USA). Dimethyl tetrachloroterephthalate (DCPA), dichlorodiphenyldichloroethylene (o,p'-DDE and p,p'-DDE), dichlorodiphenyltrichloroethane (o,p'-DDT and p,p'-DDT), and endosulfan II were purchased from Thermo Fisher Scientific (Massachusetts, USA). 
Labeled standards d14-trifluralin, d6-alpha-HCH, d8-p,p'-DDT and d4-alpha-endosulfan were obtained from CDN Isotope Inc. (Quebec, Canada). d10-chlorpyrifos was purchased from SigmaAldrich, Inc. (Missouri, USA).

\subsection{Leaf Collection and Preparation}

Alfalfa and citrus leaves were collected by hand from live plants growing in a hay-production farm in Utah and a grove in Florida, respectively. Leaf samples were homogenized and crushed into a powder using a mortar and pestle and $\sim 400 \mathrm{~mL}$ of liquid nitrogen per sample. Crushed leaves were stored in glass jars at $-20{ }^{\circ} \mathrm{C}$ until analysis. Leaf homogenates $(0.5 \mathrm{~g})$ were mixed with $1.5 \mathrm{~g} \mathrm{DE}$ to absorb moisture. When conducting the Layered ASE and Layered EDGE methods, extraction cells were packed (from bottom to top) with distinct layers of selected amounts of Florisil, GCB, and leaf/DE homogenate. When conducting Mixed ASE and Mixed EGDE methods, selected amounts of sorbents were mixed into the leaf/DE homogenate with a mortar and pestle before being packed into cells.

\subsection{Lipid Mass Determination}

The total extracted lipid masses in alfalfa and citrus leaves were determined by extracting them in the absence of sorbents using the solvents and parameters outlined in the ASE and EDGE Method sections. The mass of extracted lipids was determined gravimetrically by transferring the extract to a pre-weighed aluminum weigh boat and allowing the solvent to evaporate. The aluminum weigh boat was then placed in a $105^{\circ} \mathrm{C}$ oven overnight and its mass was determined the next day.

Residual lipid masses were measured for each test conducted during the in-cell cleanup optimization procedure for the Layered ASE method with alfalfa leaves and eventually, for each leaf type with the Layered ASE, Mixed ASE, Layered EDGE, and Mixed EDGE methods. We 
note that non-volatile chemicals other than lipids may have also been extracted and measured during this procedure. Efforts were not made to determine the identity of extracted non-volatile chemicals since any of them can present problems during gas chromatography (GC) analysis.

\subsection{UV-Visible Spectra of the Leaf Extracts}

UV-Vis absorbance spectra were obtained using an Ocean Optics (Florida, USA) USB-ISSUV/VIS. The absorbance spectra were collected from 200-890 nm using an integration time of $100 \mathrm{~ms}$ and 10-scan averaging. Instrument reproducibility was evaluated by analyzing an extract on two different days. To evaluate reproducibility of UV-Vis spectra for different extractions of the same leaf sample, two portions of a homogenized alfalfa sample were extracted separately and then analyzed. Good agreement was observed in both cases (Figure S1 and S2). UV-Vis spectra of extracts were obtained for each leaf type and on each instrument with (a) no sorbent, (b) with 5 $\mathrm{g}$ of Florisil, and (c) with $5 \mathrm{~g}$ of Florisil and $0.6 \mathrm{~g}$ of GCB added to the extraction cell.

\subsection{Spike and Recovery Experiments}

Spike and recovery experiments were conducted by spiking $15 \mu \mathrm{L}$ of a solution containing $8 \mathrm{ng} / \mu \mathrm{L}$ of each target pesticide into extraction cells prepared according to the Layered ASE, Mixed ASE, Layered EDGE, and Mixed EDGE methods. The target pesticide solution was added directly to the sample after it was introduced into the extraction cells to minimize potential volatilization losses during the transfer. Each time that cells were spiked, a spike control was prepared by spiking the same volume of target pesticide solution into $300 \mu \mathrm{L}$ of ethyl acetate. Extracts were blown down to $300 \mu \mathrm{L}$ and placed in a $\mathrm{GC}$ vial containing a $400 \mu \mathrm{L}$ insert. $15 \mu \mathrm{L}$ of a solution containing $8 \mathrm{ng} / \mu \mathrm{L}$ of each isotopically labelled compound was added to each extract and spike control. The isotopically labelled compounds were used as internal standards to account for instrument 
variability. All spike and recovery experiments were conducted in triplicate. Analyte concentrations in leaf samples that had not been spiked were independently measured and subtracted before the percent recovery was calculated.

\subsection{ASE Methods}

All ASE experiments were conducted with an ASE-350 (Thermo Fisher Scientific, Massachusetts, USA) using 25:75 (v/v) ethyl acetate: $n$-hexane and the following parameters: 5 min heat time, 10 min static time, $50 \%$ solvent flush, 3 static cycles, $120 \mathrm{~s}$ purge, $80{ }^{\circ} \mathrm{C}$ and $1500 \mathrm{psi}$. This solvent combination and set of ASE parameters were based off those used by Perez et al. [16], but changes were made based on preliminary experiments.

In Test 1 of the Layered ASE method optimization procedure for alfalfa leaves, 34-mL ASE extraction cells were packed (from bottom to top) with a cellulose filter, $10 \mathrm{~g}$ of Florisil, the leaf/DE homogenate, sand to fill the pore space, and a second cellulose filter. In Test 2, cells were packed (from bottom to top) with a cellulose filter, $5 \mathrm{~g}$ of Florisil, $0.3 \mathrm{~g}$ of GCB, a second cellulose filter, the leaf/DE homogenate, sand to fill the pore space, and a third cellulose filter paper. In Test 3, the amount of GCB was increased to $0.6 \mathrm{~g}$. The Layered ASE method was next tested with citrus leaves and then modified to produce the Mixed ASE method, which was tested on both alfalfa and citrus leaves.

\subsection{EDGE Methods}

An EDGE application note for pesticides suggests using acetonitrile as the extraction solvent, cellulose C4 Q-Disks, and the following parameters: $20 \mathrm{~mL}$ top volume, $10 \mathrm{~mL}$ bottom volume, $100{ }^{\circ} \mathrm{C}$, a 1 -min hold time, and two wash cycles ( $30 \mathrm{~mL}$ of water followed by $10 \mathrm{~mL}$ of acetonitrile) [17]. To facilitate comparison of ASE and EDGE, we adapted this method such that it incorporated 
several of the parameters from the Layered ASE method. Thus, our Layered EDGE method used 25:75 (v:v) ethyl acetate: $n$-hexane, C4 Q-Disks, and the following parameters: $20 \mathrm{~mL}$ top volume, $10 \mathrm{~mL}$ bottom volume, $80^{\circ} \mathrm{C}$, a 3-min hold time, and three wash cycles $(10 \mathrm{~mL}$ of $25: 75$ (v:v) ethyl acetate: $n$-hexane in each cycle). In the Layered EDGE method, cells were packed with a C4 Q-Disk followed by a layer of $5 \mathrm{~g}$ of Florisil, a layer of $0.6 \mathrm{~g}$ of GCB, and a top layer of the leaf/DE homogenate. This method was tested with both alfalfa and citrus leaves and then modified to produce the Mixed EDGE method, which was also tested with both alfalfa and citrus leaves.

\subsection{Comparison of Pesticide Concentrations in Field Samples}

Pesticide concentrations obtained in alfalfa and citrus field samples using each of the four methods (Layered ASE, Mixed ASE, Layered EDGE, and Mixed EDGE) were compared. In these experiments, packed extraction cells were spiked with $15 \mu \mathrm{L}$ of a solution containing $8 \mathrm{ng} / \mu \mathrm{L}$ of each isotopically labelled compound. In this case, labelled compounds were used as pesticide 'surrogates' since they underwent all sample preparation and analysis steps. All experiments were conducted in triplicate.

\subsection{Quantification of Pesticides in Leaf Extracts by GC-MS/MS}

All extracts were reduced in volume to $300 \mu \mathrm{L}$ using a Turbovap II (Biotage, North Carolina, USA) with a water bath temperature of $35^{\circ} \mathrm{C}$ and a starting flow rate of $3.0 \mathrm{~L} / \mathrm{min}$, ramp to 5 $\mathrm{L} / \mathrm{min}$ over 10 minutes, ramp to $6.0 \mathrm{~L} / \mathrm{min}$ over 5 minutes, hold at $6.0 \mathrm{~L} / \mathrm{min}$ until extracts reached $300 \mu \mathrm{L}$. Extracts were then transferred to GC vials. Pesticides were quantified with a Thermo Fisher Scientific (Massachusetts, USA) Trace 1310 GC and TSQ 8000 Evo mass spectrometer (MS). Target analytes were separated with a $30 \mathrm{~m} \times 0.25 \mathrm{~mm} \times 0.25 \mu \mathrm{m}$ ZB-5MSplus fused silica capillary column (Phenomenex, California, USA) with a 10-m deactivated guard column (Thermo 
Fisher Scientific, Massachusetts, USA). The inlet temperature was $300{ }^{\circ} \mathrm{C}$ and injections were conducted in splitless mode. The oven temperature program was: $90{ }^{\circ} \mathrm{C}$ (hold $0.5 \mathrm{~min}$ ), ramp to $170{ }^{\circ} \mathrm{C}$ at $15^{\circ} \mathrm{C} / \mathrm{min}$, ramp to $210{ }^{\circ} \mathrm{C}$ at $1{ }^{\circ} \mathrm{C} / \mathrm{min}$, ramp to $300{ }^{\circ} \mathrm{C}$ at $5{ }^{\circ} \mathrm{C} / \mathrm{min}$ (hold $10 \mathrm{~min}$ ). The MS was operated in electron ionization-selective reaction monitoring (EI-SRM) mode using argon as the collision gas. Target analyte retention times and SRM transitions are provided in Table S2. Calibration curves were prepared using the ratio of the target analyte peak area to the corresponding surrogate peak area.

\subsection{Quality Assurance}

Sand blanks and empty cells were extracted on the ASE and EDGE, respectively, before every set of samples to minimize risk of carryover. Laboratory blanks, which were prepared by packing extraction cells with all components except leaf/DE homogenate, were processed using the same methods as samples and were extracted with every batch of ASE and EDGE samples. Target analyte concentrations were only reported if the concentrations were 2.5 times that in the laboratory blank. Additionally, a check standard was run after every 4-6 extracts on the GCMS/MS to monitor the robustness of the calibration curve and instrument variability.

\subsection{Statistical Analysis}

Student t-tests were performed using Microsoft Excel 2016. ANOVA analyses were conducted using a Tukey Test using $\mathrm{R}$ version 3.6.0. Comparisons were considered significantly different if $p<0.05$.

\section{Results and Discussion:}

\subsection{Optimizing the Layered ASE Method}


The alfalfa extracted using the ASE parameters when no sorbent was included in the extraction

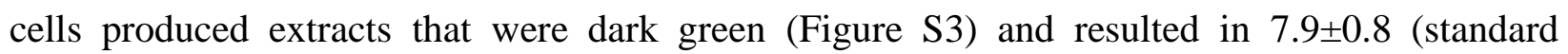
deviation) $\mathrm{mg}$ of total extracted lipid per 0.5 -g leaf sample (Figure 1). Test 1, which incorporated $10 \mathrm{~g}$ of Florisil layered below the sample, resulted in six times less extracted lipid in the sample extract, or $1.4 \pm 0.7 \mathrm{mg}$ of residual lipid. Nonetheless, Test 1 extracts were still excessively colored for GC analysis (Figure S3). Thus, in Test 2 we incorporated $0.3 \mathrm{~g}$ GCB since GCB had previously been shown to remove colored plant pigments from extracts during SPLE [16]. We also used less Florisil $(5 \mathrm{~g})$ in Test 2 because the residual lipid mass was already below our criterion of $10 \mathrm{mg}$ per extract. Test 2 extracts were still colored and contained 3.1 $\pm 1.0 \mathrm{mg}$ residual lipids. Increasing the GCB mass to $0.6 \mathrm{mg}$ in Test 3 resulted in colorless extracts (Figure S3) and $1.1 \pm 0.3 \mathrm{mg}$ of residual lipid. The spike and recovery experiment conducted with alfalfa and the Test 3 method resulted in an average percent recovery for all tested pesticides of $62 \pm 15 \%$ and average individual pesticide recoveries ranging from 37-96\% (Figure 2). While optimization of the ASE parameters (for example, by changing the extraction solvents or \% solvent flush) may have resulted in improved overall recovery, we adopted the Test 3 method as the optimized Layered ASE method. While high recoveries are ideal, the isotope dilution approach that uses labelled surrogates spiked into samples before extraction, corrects the reported concentrations for extraction inefficiencies and losses during sample preparation.

GCB has been used to reduce pigments in other extraction techniques and has commonly been used in QuEChERS [16,18-22]. In a previous study, the removal of the pigment $\beta$-carotene was monitored using UV spectra and the GCB successfully removed more than $90 \%$ of $\beta$-carotene from extracts [18]. However, other studies found that GCB does little to remove fatty acid matrix [22], so other sorbents are required to produce extracts containing minimal matrix compounds. 
This was true for our work as well; Florisil was required along with GCB to produce extracts with little residual lipid mass. While pigments have a strong affinity for GCB, some common target analytes also have an affinity for GCB. Several studies have noted that the use of more GCB leads to a reduction in extraction efficiencies for certain analytes, but for other analytes the GCB has no effect $[18,20,21,23]$.

\subsection{Extracted Lipid Masses and Extract Color}

Extracted lipid mass and extract color are two indicators of how successful the in-cell cleanup method is. While a low lipid mass and a colorless extract are desired, there is a tradeoff that may have to be made because the sorbents used for in-cell cleanup can also sorb the analytes of interest and cause a reduction in analyte recovery. Total lipid mass extracted from alfalfa using the EDGE method with no sorbents was $6.8 \pm 1.1 \mathrm{mg}$ (Figure 3 ), which is lower than that extracted with the ASE method with no sorbents, but not significantly so $(p>0.05)$. The residual lipid mass obtained from alfalfa samples using the Layered ASE method was also compared to those obtained with the Mixed ASE, Layered EDGE, and Mixed EDGE methods (Figure 3). No significant differences in residual lipid masses were observed for these four methods. In all cases, extracts were colorless and residual lipid masses were below the $10 \mathrm{mg}$ limit, indicating that extracts obtained from any of these methods are suitable for GC analysis.

When extracting total lipids from citrus leaves using the ASE method with no sorbents, $5.5 \pm 0.4$ mg lipids per 0.5-g sample were extracted (Figure 3); this is significantly lower from that obtained with alfalfa leaves using the same method. When extracting total lipids from citrus leaves using the EDGE method with no sorbents, $3.3 \pm 1.3 \mathrm{mg}$ of lipids per 0.5 -g sample were extracted from

citrus leaves, which is not significantly different from that obtained with ASE method. 
Interestingly, the reproducibility in extracted lipid quantities was worse for both alfalfa and citrus leaves when using EDGE; however, there was no indication that EDGE produced less reproducible results than ASE when all results from the study were considered.

No significant differences were observed in residual lipid masses extracted from citrus leaves when using the Layered ASE, Mixed ASE, Layered EDGE, or Mixed EDGE methods (Figure 3). The total mass extracted from the citrus with the EDGE method with no sorbents was also not significantly different from the residual masses; however, that extract was very colored (Figure S3) indicating that in-cell clean-up was needed. Extracts obtained from citrus leaves using the four in-cell clean-up methods were all colorless and contained less than $10 \mathrm{mg}$ of lipids (Figure 3), indicating that any are suitable for GC analysis.

\subsection{UV-Visible Spectra of the Leaf Extracts}

A notable difference was observed in the UV-Vis spectra of extracts obtained using the ASE methods with no sorbents versus the EDGE method with no sorbents for both alfalfa (Figure 4B versus 4D) and citrus (Figure 4C versus $4 \mathrm{E}$ ). The much stronger absorbance at $\sim 350 \mathrm{~nm}$ in the ASE extracts indicates that the EDGE method did not extract leaf matrix compounds that absorb at this wavelength to the same extent that the ASE method did. This may be advantageous for EDGE users when pesticide analysis is the objective but could be problematic if using EDGE to investigate the chemical composition of leaves.

When extracting both alfalfa and citrus leaves using the ASE and EDGE methods, the absorbance from 210-510 nm were highest in extracts obtained when no sorbent was used, less when Florisil was included, and least when Florisil plus GCB were included (Figure 4B-E). Interestingly, the peaks associated with chlorophylls A and B at $600-700 \mathrm{~nm}$ (Figure 4A) were not observed when 
Florisil was included but those at the shorter wavelengths required GCB for removal, explaining why GCB is so effective at removing green and yellow colors from extracts. The spectra in Figure 4 also show that the Layered methods were consistently more effective at removing leaf matrix compounds than the Mixed methods (both with Florisil and Florisil plus GCB methods), which has important implications for in-cell clean-up design since the effects of layering versus mixing have not been compared before.

In sum, the Layered ASE, Mixed ASE, Layered EDGE, and Mixed EDGE methods all produced extracts with low UV-Vis absorbance, providing more evidence that these methods produce extracts that will have minimal matrix interference during GC analysis. These results also suggest that UV-Vis analysis could be an effective alternative, or addition, to lipid residual mass analysis when comparing and assessing the effectiveness of various extract clean-up methods. Advantages of the UV-Vis approach over the lipid residue approach are that it provides more specific information about the relative amounts of absorbing chemicals, is considerably faster, requires less steps, and doesn't destroy the extract.

\subsection{Spike and Recovery Results}

Average recoveries for all pesticides for both types of leaves with all tested methods are shown in Figure 5. The spike and recovery results for each individual pesticide for alfalfa and citrus leaves with all tested methods are shown in Figures 2 and Figure S4-S6, respectively. The key observations from Figure 5 are that average recoveries when all pesticides were included were significantly higher when using (a) the Layered ASE method compared to the Layered EDGE method for alfalfa and (b) the Mixed ASE method compared to the Mixed EDGE method for both alfalfa and citrus. These results suggest that overall, better recoveries of spiked pesticides can be expected with ASE compared to EDGE. The key observations from the figures showing results 
for individual pesticides (Figures 2 and S4-S6) was that only a handful of the individual pesticides in each case had significantly higher recoveries with the ASE methods (Figures S7-S10); however, because most pesticides in each case had higher recoveries (even if not significantly higher) with the ASE methods, average recoveries calculated when all pesticides were included where higher with the ASE methods. For example, Figure 2 shows that with the Layered methods and alfalfa leaves, recoveries were significantly higher for only five of the 20 pesticides, but recoveries were higher (even if not significantly higher) for 16 of the 20 pesticides. The very large differences in recoveries for the pesticides with significant differences (for example, $78 \%$ versus $41 \%$ for flupyradifurone) also drove the differences in average recovery between the methods. Better recoveries of spiked pesticides with the EDGE methods may have been possible with further method development.

Significant differences in average pesticide extraction efficiencies for Layered versus Mixed methods were observed (Figure 5). Average recoveries when all pesticides were included were significantly higher when using (a) Mixed ASE method compared to the Layered ASE method for alfalfa and (b) the Layered EDGE method compared to the Mixed EDGE method for citrus leaves. These results suggest that both cell packing methods lead to similar recoveries for these pesticides. However, some compounds did show a significant difference in percent recovery (Figures S11S14). When there was a significant difference in recovery, the recovery was usually higher for the Mixed method. Recoveries may have been higher in some cases with the Mixed methods because extracted analytes did not travel through the all of the sorbent, meaning less analyte-sorbent interaction. This hypothesis is supported by the observation that the Mixed methods also generally had higher extracted lipids than the Layered method (Figures 3).

\subsection{Pesticide Concentrations in Field Samples}


The only target pesticide detected in field samples of alfalfa leaves was the pyrethroid insecticide, fenpropathrin. Its average concentration was $640 \pm 90 \mathrm{ng} / \mathrm{g}$ when all four extraction methods were considered (Figure S15). The organophosphate insecticide malathion and the pyrazole insecticide tolfenpyrad were detected in field samples of citrus leaves at average concentrations of $1290 \pm 270$ ng malathion/g citrus leaf and $140 \pm 30 \mathrm{ng}$ tolfenpyrad/g citrus leaf when all extraction methods were considered (Figure S16-S17). No significant differences in reported concentrations for any of these pesticides were observed for the four different extraction methods. More data is needed to make a broader conclusion; however, the lack of significant differences observed here suggests that ASE and EDGE methods are likely to produce similar pesticide concentrations in leaf extracts. The use of labelled surrogates increases the likelihood of this since they correct for extraction inefficiencies and losses during sample preparation.

\subsection{Method Parameter Comparison}

Several extraction parameters were compared for Layered ASE and Layered EDGE methods using alfalfa leaves (Table 1). For the lipid mass extracted, the ASE extracted more than the EDGE. This, along with the UV-Vis spectra, shows that this EDGE method did not extract as much of the leaf matrix components as the ASE did. This indicates that for the methods used in this work the EDGE has better selectivity than the ASE. This can be viewed as an advantage or disadvantage for the EDGE, depending on the goal of extraction. If the goal is to produce extracts that require less clean-up the EDGE may be a better choice, but if the goal is to extract total lipids from samples the ASE may be better suited. The residual lipid mass was similar for both the Layered ASE and Layered EDGE methods, showing that when sorbents were used for in-cell clean-up, similar amounts of leaf matrix were extracted. The average percent recovery, as well as the range, of spiked pesticides was higher with the Layered ASE method than the Layered EDGE method. 
Extraction with the ASE method used in this work took 4.5 times longer than the EDGE method. The ASE used slightly less solvent per extraction compared to the EDGE, but the amounts were similar. The ASE takes up more bench space, being almost 2 times wider than the EDGE. We also observed differences in ease of use between the instruments. The ASE methods required more time to pack the extraction cells compared to the EDGE methods. This is due to ASE cells requiring the dead space filled as well as requiring extra filters to allow for the extraction cells to be better sealed.

\section{Conclusion}

An SPLE method for extracting pesticides from leaves using the ASE was developed. The method developed led to clear colorless extracts with low residual lipid weight. An EDGE method based on the ASE method parameters was then developed. Extractions were performed for both alfalfa and citrus leaves and used sorbents in both a layered and mixed manner. The UV-Vis spectra of the Layered ASE, Mixed ASE, Layered EDGE, and Mixed EDGE methods were similar. For spike and recovery experiments, when there was a significant difference in the percent recovery, the ASE generally had the higher percent recovery. However, when extracting pesticides from field samples of alfalfa and citrus, there was no significant difference between extraction methods. The EDGE is faster than the ASE, but does use slightly more solvent. These results show that the higher pressures employed by the ASE system may result in better extraction efficiencies of both pesticides and leaf matrix compounds; however, the use of surrogates means that results in reported pesticide concentrations in field samples were very similar. It is possible that the higher pressures employed by ASE are needed for extracting more-tightly bound analytes in more complex matrices, such as fish or soil. 


\section{Acknowledgements}

We thank Autumn Slade for assistance with EDGE experiments. 


\section{References:}

[1] D. Sharp, B. Eskenazi, R. Harrison, P. Callas, A.H. Smith, Delayed health hazards of pesticide exposure, Annu. Rev. Public Health. 7 (1986) 441-471. doi:10.1146/annurev.publhealth.7.1.441.

[2] R. Rodríguez-Solana, J.M. Salgado, J.M. Domínguez, S. Cortés-Diéguez, Comparison of soxhlet, accelerated solvent and supercritical fluid extraction techniques for volatile (GC-MS and GC/FID) and phenolic compounds (HPLC-ESI/MS/MS) from lamiaceae species, Phytochem. Anal. 26 (2015) 61-71. doi:10.1002/pca.2537.

[3] K. Madej, T.K. Kalenik, W. Piekoszewski, Sample preparation and determination of pesticides in fatcontaining foods, Food Chem. 269 (2018) 527-541. doi:10.1016/j.foodchem.2018.07.007.

[4] R.B. Hoff, T.M. Pizzolato, Combining extraction and purification steps in sample preparation for environmental matrices: A review of matrix solid phase dispersion (MSPD) and pressurized liquid extraction (PLE) applications, TrAC - Trends Anal. Chem. 109 (2018) 83-96. doi:10.1016/j.trac.2018.10.002.

[5] B. Subedi, L. Aguilar, E.M. Robinson, K.J. Hageman, E. Björklund, R.J. Sheesley, S. Usenko, Selective pressurized liquid extraction as a sample-preparation technique for persistent organic pollutants and contaminants of emerging concern, TrAC - Trends Anal. Chem. 68 (2015) 119-132. doi:10.1016/j.trac.2015.02.011.

[6] M.M. Nascimento, G.O. Da Rocha, J.B. De Andrade, Pesticides in the atmospheric environment: An overview on their determination methodologies, Anal. Methods. 10 (2018) 4484-4504. doi:10.1039/c8ay01327f.

[7] P. Parrilla Vázquez, C. Ferrer, M.J. Martínez Bueno, A.R. Fernández-Alba, Pesticide residues in spices and herbs: Sample preparation methods and determination by chromatographic techniques, TrAC - Trends Anal. Chem. 115 (2019) 13-22. doi:10.1016/j.trac.2019.03.022.

[8] D. Raynie, C. Qui, Improving extraction processes and sample preparation in food analysis, LCGC North Am. 35 (2017) 158-169.

[9] H. Barchanska, M. Danek, M. Sajdak, M. Turek, Review of sample preparation techniques for the analysis of selected classes of pesticides in plant matrices, Crit. Rev. Anal. Chem. 48 (2018) 467491. doi:10.1080/10408347.2018.1451297.

[10] I. Ali, M. Suhail, O.M.L. Alharbi, I. Hussain, Advances in sample preparation in chromatography for organic environmental pollutants analyses, J. Liq. Chromatogr. Relat. Technol. 0 (2019) 1-24. doi:10.1080/10826076.2019.1579739.

[11] C.E.M. Corporation, EDGE Automated Pressurized Fluid Extraction for USEPA 3545, (2017).

[12] C.E.M Corporation, Automated Extraction System EDGE, (2017). http://cem.com/edge/ (accessed September 1, 2019).

[13] C.E.M Corporation, Application Notes, (2017). 
http://cem.com/en/literature?application=Extraction\&literature_type=Application_Notes (accessed September 1, 2019).

[14] B.E. Richter, B.A. Jones, J.L. Ezzell, N.L. Porter, N. Avdalovic, C. Pohl, Accelerated solvent extraction: A technique for sample preparation, Anal. Chem. 68 (1996) 1033-1039. doi:10.1021/ac9508199.

[15] K.S. Lavin, K.J. Hageman, Selective pressurised liquid extraction of halogenated pesticides and polychlorinated biphenyls from pine needles, J. Chromatogr. A. 1258 (2012) 30-36. doi:10.1016/j.chroma.2012.08.042.

[16] R.A. Pérez, J.L. Tadeo, B. Albero, E. Miguel, C. Sánchez-Brunete, Gas chromatography-triplequadrupole mass spectrometry for analysis of selected polyhalogenated pollutants in plants. Comparison of extraction methods, Anal. Bioanal. Chem. 405 (2013) 389-400. doi:10.1007/s00216-012-6465-x.

[17] C.E.M. Corporation, Extraction of Pesticides from Difficult Matrices, (2018) 2-4.

[18] K. Banerjee, D.P. Oulkar, S.B. Patil, M.R. Jadhav, S. Dasgupta, S.H. Patil, S. Bal, P.G. Adsule, Multiresidue determination and uncertainty analysis of 87 pesticides in mango by liquid chromatography-tandem mass spectrometry, J. Agric. Food Chem. 57 (2009) 4068-4078. doi:10.1021/jf900358r.

[19] C.M. Karbiwnyk, K.E. Miller, A reveiw of current analytical applications employing graphitized carbon black, in: I.J. Sanders, T.L. Peeten (Eds.), Carbon Black Prod. Prop. Uses, Nova Science Publishers, Inc., 2011: pp. 69-91.

[20] L. Li, W. Li, D. Qin, S. Jiang, F. Liu, Application of graphitized carbon black to the QuEChERS method for pesticide multiresidue analysis in spinach, J. AOAC Int. 92 (2009) 538-547.

[21] S. Walorczyk, Application of gas chromatography/tandem quadrupole mass spectrometry to the multi-residue analysis of pesticides in green leafy vegetables, Rapid Commun. Mass Spectrom. 22 (2008) 3791-3801. doi:10.1002/rcm.3800.

[22] F.J. Schenck, S.J. Lehotay, V. Vega, Comparison of solid-phase extraction sorbents for cleanup in pesticide residue analysis of fresh fruits and vegetables, J. Sep. Sci. 25 (2002) 883-890. doi:10.1002/1615-9314(20021001)25:14<883::AID-JSSC883>3.0.CO;2-7.

[23] B. Łozowicka, P. Mojsak, P. Kaczyński, R. Konecki, A. Borusiewicz, The fate of spirotetramat and dissipation metabolites in Apiaceae and Brassicaceae leaf-root and soil system under greenhouse conditions estimated by modified QuEChERS/LC-MS/MS, Sci. Total Environ. 603-604 (2017) 178184. doi:10.1016/j.scitotenv.2017.06.046.

[24] S. Prahl, Chlorophyll a, (2017). https://omlc.org/spectra/PhotochemCAD/html/123.html (accessed December 4, 2019).

[25] S. Prahl, Chlorophyll b, (2017). https://omlc.org/spectra/PhotochemCAD/html/125.html (accessed December 4, 2019). 


\section{Figure Captions}

Figure 1. Comparison of the lipid mass extracted for each step of the method development process for $0.5 \mathrm{~g}$ alfalfa leaves on the ASE. Method development samples were packed in a layered manner. In addition to the $0.5 \mathrm{~g}$ alfalfa leaves, Test 1 cells contained $10 \mathrm{~g}$ of Florisil, Test 2 cells contained $5 \mathrm{~g}$ of Florisil and $0.3 \mathrm{~g} \mathrm{GCB}$, and Test 3 cells contained $5 \mathrm{~g}$ of Florisil and $0.6 \mathrm{~g}$ GCB. Each test was conducted in triplicate and error bars indicate standard deviation. Different letters above the bars indicate a significant difference.

Figure 2. Comparison of the percent recoveries from alfalfa leaves using both the Layered ASE and Layered EDGE methods. The boxes surrounding compounds indicate that the percent recovery from the two methods are significantly different. Each test was conducted in triplicate and error bars indicate standard deviation.

Figure 3. Lipid mass and residual lipid mass extracted for each extraction method when using no sorbents and when using the Layered ASE, Mixed ASE, Layered EDGE, and Mixed EDGE methods. Each test was conducted in triplicate and error bars indicate standard deviation. Different letters above the bars indicate a significant difference.

Figure 4. UV-Vis absorbance spectra for extracts from each method. A) Reference UV-Vis spectrum for a plant extract in diethyl ether showing expected absorbance for chlorophyll a and chlorophyll b [24,25]. B) - E) UV-Vis spectra for alfalfa and citrus leaves extracted with no sorbent, only Florisil, and using the Layered ASE, Mixed ASE, Layered EDGE, and Mixed EDGE methods.

Figure 5. Comparison of the average percent recoveries when taking all compounds into account when using the Layered ASE, Mixed ASE, Layered EDGE, and Mixed EDGE methods. Each test was conducted in triplicate and error bars indicate standard deviation. Different letters above the bars indicate a significant difference. 